\title{
Morphology of Single Geniculocortical Afferents and Functional Recovery of the Visual Cortex after Reverse Monocular Deprivation in the Kitten
}

\author{
Antonella Antonini, Deda C. Gillespie, Michael C. Crair, and Michael P. Stryker \\ W. M. Keck Foundation Center for Integrative Neuroscience, Department of Physiology, University of California, San \\ Francisco, California 94143-0444
}

To investigate the possible anatomical basis for the functional recovery of visual cortical responses after reverse monocular deprivation, we have studied the morphology of single geniculocortical afferents to area 17. In kittens reverse-sutured for $10 \mathrm{~d}$ after an initial week of monocular deprivation, single-unit and intrinsic signal optical recordings confirmed that the effects of the initial deprivation were largely reversed. Responses through the originally nondeprived (OND) eye were drastically diminished, but remained much more selective for orientation than after an initial monocular deprivation (Crair et al., 1997). Responses through the originally deprived (OD) eye recovered completely. Geniculocortical afferent arbors in layer IV of area 17 were filled by iontophoresis of Phaseolus lectin into lamina A of the lateral geniculate nucleus (LGN) and were serially reconstructed. Arbors serving both the OD and the OND eye were

In many species of mammals, closure of one eye during a sensitive period in early postnatal development leads to a loss of normal binocular interactions in the visual cortex, and the vast majority of neurons lose their responses to the closed, deprived eye (Wiesel and Hubel, 1963, 1965; Hubel and Wiesel, 1970; Shatz and Stryker, 1978; LeVay et al., 1980; Sherman and Spear, 1982; Fregnac and Imbert, 1984; Mitchell, 1991). However, a substantial functional recovery of cortical responses through the deprived eye can be obtained by forcing the use of the deprived eye by patch therapy or reverse lid suture. In this experimental paradigm, after the initial period of monocular deprivation (MD), the eye that was originally deprived (OD) is reopened and allowed normal vision, whereas the originally nondeprived (OND) eye is sutured closed (Hubel and Wiesel, 1970; Chow and Stewart, 1972; Blakemore and van Sluyters, 1974; Movshon, 1976; Mitchell et al., 1977; Olson and Freeman, 1977; Blakemore et al., 1978, 1981; Giffin and Mitchell, 1978).

In the cat, the rate and extent of the physiological changes produced by reverse suture depend on the age of the animal and the length of the original deprivation (Blakemore and van

\footnotetext{
Received July 7, 1998; revised Sept. 14, 1998; accepted Sept. 15, 1998.

This work was supported by National Institutes of Health Grant EY02874 to M.P.S.

Correspondence should be addressed to Dr. Michael P. Stryker, Department of Physiology, Room S-762, Box 0444, 513 Parnassus Avenue, University of California, San Francisco, CA 94143-0444.

Dr. Gillespie's present address: Department of Neurobiology, Northwestern University, Evanston, IL 60208.

Dr. Crair's present address: Department of Neuroscience, Baylor College of Medicine, Houston, TX 77030.

Copyright (C) 1998 Society for Neuroscience $\quad 0270-6474 / 98 / 189896-14 \$ 05.00 / 0$
}

analyzed. The plastic changes of both OD and OND arbors were evaluated by comparison with arbors reconstructed in normal animals and in animals studied after an equivalent initial period of deprivation (Antonini and Stryker, 1996). These analyses demonstrate that closure of the OND eye caused a substantial shrinkage of the arbors serving that eye. Moreover, reopening the OD eye induced regrowth only in some arbors, whereas others appeared to be largely unaffected and continued to have the characteristics of deprived arbors. Quantitatively, the initial and the second deprivation caused similar proportional changes in total arbor length and numbers of branches, whereas several other features were more severely affected by the initial deprivation.

Key words: area 17; reverse suture; axonal reconstruction; optical imaging; critical period; monocular deprivation

Sluyters, 1974; Movshon and Blakemore, 1974; Dursteler et al., 1976; Movshon, 1976; Giffin and Mitchell, 1978; van Sluyters, 1978; Blakemore and Hawken, 1982; Malach et al., 1984). At the peak of the sensitive period, it is possible to reverse completely the cortical ocular dominance to favor the OD eye, even when the initial MD was initiated at eye-opening.

At present, we have some understanding of the anatomical changes accompanying the physiological effects induced by the initial MD. These include a substantial loss of geniculocortical terminals serving the deprived eye in layer IV of the visual cortex (Hubel et al., 1977; Shatz and Stryker, 1978; LeVay et al., 1980; Antonini and Stryker, 1993a, 1996) if the deprivation period lasts for 1 week or more. However, an expansion of the nondeprived terminals was observed only in animals deprived for many weeks starting from eye-opening and not in animals deprived for 1 week around the middle of the critical period (LeVay et al., 1980; Antonini and Stryker, 1993a, 1996). Together, these results suggest that the processes responsible for new growth of terminals are slower than those responsible for their removal. All of these anatomical changes appear to take place more slowly than the effect of MD on the physiological reorganization of cortical responses, in which the loss of response to the deprived eye is almost saturating with $2 \mathrm{~d}$ of deprivation (Movshon and Dursteler, 1977; Freeman et al., 1981; Crair et al., 1997).

The present experiments were undertaken to determine whether the loss of function of the OND eye and the complementary recovery of responses to the OD eye after reverse suture can be attributed to anatomical changes-the pruning and reexpansion of geniculocortical connections serving the two eyes. Indeed, a partial new growth of connections serving the OD eye 
has already been suggested by transneuronal experiments in monkeys that were reverse-sutured after several weeks of MD (LeVay et al., 1980; Swindale et al., 1981). These experiments show geniculocortical terminals serving the OD eye in regions of layer IV that were devoid of afferent connections after the initial MD.

To study the anatomical basis of the functional recovery, we have serially reconstructed single geniculocortical arbors in reverse-sutured animals in which the length of the first MD and the age at which it was performed were equivalent to those used earlier to study the effects of an initial period of MD (Antonini and Stryker, 1993b, 1996). The anatomical data are preceded by an analysis of the efficacy of the particular protocol of reverse suture used in these experiments at restoring functional cortical responses, using intrinsic signal optical imaging and single-unit recording.

\section{MATERIALS AND METHODS}

Seven normally pigmented kittens were used for this study. All kittens were born at the University of California San Francisco cat colony and were kept with their mother throughout the experiment. Six kittens underwent the reverse-suture manipulation, and one was used as an age-matched control. Among the reverse-sutured animals, two were used exclusively for optical imaging of the visual cortex and single unit recordings, three were used exclusively for single arbor reconstruction, and finally, one was used for both the anatomical and physiological experiments (see Tables 1,2).

\section{Reverse suture}

The experimental paradigm consisted of performing MD for 6 or $7 \mathrm{~d}$ during the fifth week of age (first MD) followed by a reverse suture of the other eye for a subsequent 10 or $11 \mathrm{~d}$ period (second MD). Tables 1 and 2 list for each kitten the age at the time of each procedure and the eye involved in the first or second MD. Monocular deprivations were performed under halothane anesthesia $(1-2 \%)$ in $\mathrm{N}_{2} \mathrm{O} / \mathrm{O}_{2}(1: 1)$. For the first $\mathrm{MD}$, the superior and inferior eyelids were joined along the margins and sutured with sterile surgical \#4 Vicryl (Ethicon, Somerville, NJ). Ophthalmic glue (Nexaband, Phoenix, AZ) was applied to both of the eyelid margins and to the Vicryl stitches to prevent reopening of the eye. For the second $\mathrm{MD}$, the eyelids were trimmed along the margins and then sutured with sterile surgical \#4 Vicryl. In both procedures, topical antibiotic ointment was applied to the eye before the eyelids were sutured. A small opening on the nasal end of the eyelid allowed lachrymal drainage. Antibiotics were administered systemically, and the animals were checked once or twice a day to ensure that no openings formed along the eyelids.

\section{Optical imaging and single unit recordings}

The animal was initially anesthetized with halothane mixed with $\mathrm{N}_{2} \mathrm{O} / \mathrm{O}_{2}$ (1:1). This initial anesthesia permitted the insertion of a catheter into the femoral vein for subsequent drug delivery and intubation of the trachea for artificial ventilation. The animal was then positioned on the HorsleyClarke stereotaxic apparatus. The electrocardiogram, expired $\mathrm{CO}_{2}(3.8-$ $4.2 \%$ ), and rectal temperature were monitored continuously. Anesthesia was maintained throughout the experiment by infusion of sodium thiopental $(10 \mathrm{mg} / \mathrm{ml}$; Abbott, North Chicago, IL) followed by pentobarbital $(10 \mathrm{mg} / \mathrm{ml}$; Abbott). Muscle paralysis was induced with a bolus of gallamine triethiodide $(1.5 \mathrm{mg} / \mathrm{kg}$; Sigma, St. Louis, MO) and maintained by continuous infusion of the same drug $\left(1 \mathrm{mg} \cdot \mathrm{kg}^{-1} \cdot \mathrm{h}^{-1}\right.$ in $2.5 \%$ dextrose/lactate Ringer's solution). Throughout the experiment, the level of anesthesia was assessed by continuously monitoring the heart rate. Additional thiopental was given to maintain the heart rate at or below its level during the preparatory phase of the experiment, when the animal was intubated and positioned in the stereotaxic apparatus but was not paralyzed and could therefore react to noxious stimuli.

Details of the optical imaging protocols have been published previously (Crair et al., 1997). Briefly, a bone flap of approximately $10 \mathrm{~mm} \times$ $10 \mathrm{~mm}$ was removed above area 17, around anteroposterior 0 of the stereotaxic coordinates. The dura was carefully removed, and the opening was filled with $3 \%$ agarose and sealed with a clear glass coverslip to ensure a flat surface. The cortical surface was illuminated with a green light, and the slow-scan CCD camera (Princeton Instruments, Trenton, $\mathrm{NJ}$ ) was initially focused on the pial surface to obtain a clear image of the blood vessels. For acquiring intrinsic signals related to cortical activity, the surface of the cortex was illuminated with $610 \mathrm{~nm}$ light, and the camera was focused $250-500 \mu \mathrm{m}$ below the pial surface. The animal was visually stimulated with computer-generated square-wave gratings $(0.10$ or 0.15 cycles/degree) moving in both directions at four different orientations $\left(0,45,90\right.$, and $\left.135^{\circ}\right)$. The stimuli were presented on a monitor placed $40 \mathrm{~cm}$ in front of the animal. For each run, the four oriented stimuli and a blank screen isoluminant with average grating luminance were presented to each eye individually; these presentations were repeated 16 times in random order. For each stimulus orientation, the acquired raw images were averaged and then normalized against the blank gray stimulus. Images were processed with 3-pixel low-pass and 70-pixel high-pass filters (each pixel in the final images occupied $\sim 17 \mu \mathrm{m}$ on the cortex). Angle maps were constructed by determining, for each pixel, the orientation that gave the best signal, and assigning a color to each orientation. An overall optical Contralateral Bias Index [optical CBI; Crair et al. (1998)] was calculated for each map by assigning to each pixel an eye dominance value based on the relative response strength through each eye and then averaging all pixels within the regions of the maps that were free of artifact. CBI values of 1.00 and 0.00 represent complete dominance of the contralateral eye and the ipsilateral eye, respectively.

Once the optical imaging was completed, the coverslip and the top layer of the agarose were removed, and the activity of single units was recorded extracellularly with tungsten electrodes that were advanced vertically through the medial bank of the lateral gyrus, at $\sim 0$ of the anterior-posterior stereotaxic coordinates. Visual stimuli consisted of oriented light bars moved by hand in different directions. Neurons were classified in the seven traditional ocular dominance groups according to Hubel and Wiesel (1962).

Two indices were used to evaluate the ocular preference. The "reversal index" (RI), which simply represents the proportion of neurons dominated by the OD eye, has been used in previous works (Blakemore and van Sluyters, 1974; Movshon, 1976) to estimate the extent of the ocular dominance shift reversal and is used here for a comparison between our data and those published previously. In our experiments the OD eye was the right eye, and we have recorded in all cases from the right hemisphere. Therefore, the reversal index is given by the sum of neurons in classes 5,6 , and 7 divided by the total number of neurons. The CBI is a weighted average toward one or the other eye and is calculated by the formula: $\mathrm{CBI}=[(n 1-n 7)+2 / 3(n 2-n 6)+1 / 3(n 3-n 5)+N] / 2 N$, where $N$ is the total number of cells and $n x$ is the number of cells in a ocular dominance group $x$. CBI values of 1.00 and 0.00 represent complete dominance by the contralateral or the ipsilateral eye, respectively. This index is analogous to the optical CBI.

\section{PHA-L experiments}

Single geniculocortical arbors, anterogradely labeled with the Phaseolus lectin PHA-L (Vector, Burlingame, CA) (Gerfen and Sawchenko, 1984) injected into the LGN, were serially reconstructed in one control and four reverse-sutured animals. Table 2 lists the entire sample of geniculocortical arbors reconstructed in the two groups of animals.

\section{PHA-L injections}

The goal of the experiment was to inject PHA-L into lamina A of the right and left LGN, allowing the analysis, in the same animal, of geniculocortical arbors serving the OD and OND eyes. Thus, special care was taken to clearly identify electrophysiologically the main laminae of the LGN before the PHA-L injection. Electrophysiological recordings and PHA-L injections into the LGN were performed according to the protocol described in detail in previous papers (Antonini and Stryker, 1993b, 1996). Briefly, the animal was initially anesthetized with a mixture of ketamine hydrochloride $(0.2 \mathrm{mg} / \mathrm{kg}$; Ketalar, Parke-Davis, Morris Plains, NJ) and acepromazine $(0.02 \mathrm{mg} / \mathrm{kg}$; PromAce, Ayerst, Rouses Point, $\mathrm{NY})$. Anesthesia was maintained with halothane (1-2\%) in $\mathrm{N}_{2} \mathrm{O} / \mathrm{O}_{2}(1: 1)$ throughout the experiment. Tungsten microelectrode penetrations were made between the Horsley-Clarke stereotaxic coordinates anteroposterior 4.0-6.0 and mediolateral 7.0-8.5. Once a clear response from lamina A was obtained, the metal microelectrode was withdrawn and substituted with a PHA-L-filled glass pipette $10-15 \mu \mathrm{m}$ in diameter. The $2.5 \%$ solution of PHA-L (Vector) in $0.1 \mathrm{~m}$ sodium PBS, $\mathrm{pH} 8$, was 
iontophoretically injected using a high-voltage current source device (Midgard Electronics; Stoelting, Wood Dale, IL) with a positive current ( $8 \mu \mathrm{Amp})$ delivered in $7 \mathrm{sec}$ pulses for $4 \mathrm{~min}$. Usually, two to four lectin injections were made in each LGN. After completion of the experiment the skin of the skull was sutured closed, and antibiotic was administered systemically. PHA-L was injected at the end of the first period of MD; the deprived eye was reopened to allow electrophysiological recordings from both LGNs. At the end of the procedure the OND eye was then sutured closed.

\section{Perfusion and immunohistochemistry}

Ten days after the PHA-L injections, equivalent to $10 \mathrm{~d}$ of reverse suture, the animals were killed with an overdose of Nembutal $(60 \mathrm{mg} / \mathrm{kg})$ and perfused transcardially with, in succession, ice-cold $0.1 \mathrm{M}$ phosphate buffer; $4 \%$ paraformaldehyde, $0.5 \%$ glutaraldehyde in $0.1 \mathrm{~m}$ phosphate buffer; $4 \%$ paraformaldehyde, $3.42 \mathrm{gm} / 1$ L-lysine (Sigma), $0.55 \mathrm{gm} / 1$ sodium meta-periodate (Sigma) in $0.1 \mathrm{M}$ phosphate buffer. All solutions had a $\mathrm{pH}$ of 7.4. The brain was removed and post-fixed overnight. Blocks containing the LGN and the entire caudal pole of each hemisphere where the visual cortex is located were embedded in gelatin-albumin and cut $(80 \mu \mathrm{m})$ with a vibratome in the frontal plane. All sections were

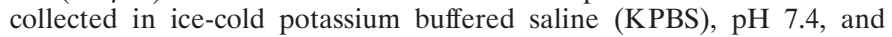
processed for standard indirect immunohistochemistry. The immunohistochemical procedure has been described in detail in previous papers (Antonini and Stryker, 1993a, 1996). Briefly, sections were incubated overnight at $4^{\circ} \mathrm{C}$ in a blocking solution composed of $2.5 \%$ bovine serum albumin (BSA), $2 \%$ normal rabbit serum (NRS), and $0.7 \%$ Triton X-100. They were then transferred into a solution of $2.5 \%$ BSA and $2 \%$ NRS containing the primary antibody (goat anti-PHA-L, Vector) at a dilution of $1: 1000$ and kept at $4^{\circ} \mathrm{C}$ for $48-72 \mathrm{hr}$. Sections were subsequently incubated overnight at $4^{\circ} \mathrm{C}$ in a solution containing rabbit anti-goat biotinylated secondary antibody (Vector) in 2.5\% BSA, 2\% NRS, and $0.3 \%$ Triton X-100, then transferred for 3-4 hr in an avidin-HRP complex, and finally reacted with a solution of $0.05 \%$ diaminobenzidine hydrochloride (DAB) (Sigma), 0.7\% nitroammonium sulfate, and 0.3\% hydrogen peroxide in Tris buffer, $\mathrm{pH}$ 7.4. All of the washes between the incubation in the primary and secondary antibody and between the secondary antibody and the avidin-HRP complex solution were performed in KPBS; the final washes before and after the DAB reaction were made in Tris buffer.

\section{Axonal reconstructions}

We considered for analysis only those experiments in which PHA-L injection sites were limited to the binocular zone of the LGN and confined to layer A. We limited our analysis to axons with well filled terminal arborizations that could be followed up to their thinnest terminals.

PHA-L-filled arbors were three-dimensionally reconstructed at $1000 \times$ from serial sections with the aid of a computer graphic system, the Neurotrace system (InterAction, Boston, MA), which has been described previously (Passera et al., 1988; Antonini and Stryker, 1993b, 1996). In Figures 3-5, the axonal reconstructions are shown in two views: in the coronal plane (as reconstructed) and after rotation about the ventrodorsal axis, providing in this case a view tangential to the pial surface.

Several measurements were used to quantify the features of the terminal arborization of the reconstructed afferents [also see Antonini and Stryker (1993b, 1996)].

Measurements related to the size of the geniculocortical arbors: the total linear length of the arborization. This value was obtained by the addition of the lengths of all the branches constituting the terminal field of an arbor. Only the portion of the arbor located in layers III and IV was considered for this measure. For this reason, the main axonal trunk was clipped just below layer IV, as is clearly visible in arbors shown in Figures $3-5$. Terminal branches $<5 \mu \mathrm{m}$ were not considered for the analysis.
Measurements related to the size of the geniculocortical arbors: the coverage area of the arborization in layer $I V$. The terminal arborization was considered to be compressed along an axis perpendicular to the pial surface and to lie in a single plane. From this view, the coverage area of the arbor was calculated as the area over which arbor density (see below) was higher than $2 \mu \mathrm{m} / 1000 \mu \mathrm{m}^{2}$.

Measurements related to the complexity and density of the geniculocortical arbors: the number of branch points of the terminal arborization in layer $I V$. Again, branch points giving rise to terminals $<5 \mu \mathrm{m}$ in length were not considered.

Measurements related to the complexity and density of the geniculocortical arbors: the density of the terminal arborization in layer IV. For comparison with earlier findings (Antonini and Stryker, 1993b), we measured the density of axonal branches per unit area within layers III/IV rather than the density of synaptic boutons. The arbor density measure used here is not directly related to synaptic density, because some thick axonal branches may bear only a small number of boutons. Density was evaluated from the two-dimensional pial view of the arbor, after compression along an axis perpendicular to the pial surface. The density at each $5 \times 5 \mu \mathrm{m}$ square within the territory covered by the arbor was calculated by summing the total lengths of the portions of all branches that lay within the area enclosed by a circle of $100 \mu$ m diameter. The maximal density of the terminal arborization (expressed in micrometers per $1000 \mu \mathrm{m}^{2}$ ) was then evaluated.

Measurements related to the complexity and density of the geniculocortical arbors: high-density areas of the terminal arborization. With the aim of better understanding the internal organization of the terminal arborization, we used this measure to reveal the presence of areas of dense clustering of collateral branches. In a previous paper (Antonini and Stryker, 1993b) we defined the standard characteristics of zones of high density of innervation, called "patches," from the analysis of arbors reconstructed in normal animals at postnatal day (P) 30/31 and P40, which is the same population used here as control. For each arbor, the high-density patches were defined as regions of the terminal arborization exceeding a threshold density of half the maximal density. The average threshold density across animals was $38 \mu \mathrm{m}$ of arbor length per $1000 \mu \mathrm{m}^{2}$ of area and was used to define the threshold density for a standard cluster. For each arbor, we summed the areas of the individual patches to obtain the total area of the high-density clusters.

For all features measured, evaluation of the differences among groups has been obtained by comparing groups two at a time, using the MannWhitney $U$ test for nonparametric statistical analyses.

\section{RESULTS}

The results are presented in three sections. First, we describe the physiological characteristics of the visual cortex in reversesutured animals, based on the analysis of both optical imaging of intrinsic signals in area 17 and single-unit recordings. Next, we describe and compare serially reconstructed geniculocortical arbors serving the OD and OND eyes in reverse-sutured animals, along with arbors reconstructed in an age-matched control. Finally, we present measurements of the size, complexity, and distribution of the reconstructed arbors and compare the effects of reverse suture with those of an initial period of monocular deprivation.

\section{Ocular dominance and response characteristics in area 17 of reverse-sutured animals}

In three animals (K2, K12, and K28) (Table 1), optical imaging of intrinsic signals was acquired from a $2.4 \times 3.2 \mathrm{~mm}$ area centered

\section{Table 1. Experimental protocol for animals used for optical imaging and single unit recordings}

\begin{tabular}{|c|c|c|c|c|c|c|}
\hline \multirow[b]{2}{*}{ Animals } & \multicolumn{2}{|c|}{ First deprivation } & \multicolumn{2}{|c|}{ Second deprivation } & \multirow[b]{2}{*}{ Age at death } & \multirow[b]{2}{*}{ Recorded hemisphere } \\
\hline & Eye & Age & Eye & Age & & \\
\hline $\mathrm{K} 2$ & Right & P33 & Left & P40 & P50 & Right visual cortex \\
\hline K12 & Right & P33 & Left & $\mathrm{P} 40$ & P50 & Right visual cortex \\
\hline $\mathrm{K} 28$ & Right & P32 & Left & P39 & P49 & Right visual cortex \\
\hline
\end{tabular}


on the lateral gyrus over the intra-aural line. In all three animals, the originally deprived eye was on the right, and the recordings, after the end of the reverse-suture period, were obtained from the right hemisphere. Figure $1 A$ shows for the reverse-sutured kitten K12 the distribution of cortical activity in response to the four oriented stimuli (see Materials and Methods) presented to the OD $(a-d)$ and OND $(e-h)$ eye. The cortical activity elicited through the OD eye was strong and selective for each stimulus orientation, indicating that the pathway driven by this eye had fully recovered within the $10 \mathrm{~d}$ of reverse suture. The activity map driven by the OND eye showed a much weaker signal, but the responses through this eye remained clearly orientation selective. Figure $1 B$ shows the effect of the initial period of deprivation, before the reverse suture, in a P38 animal monocularly deprived for $7 \mathrm{~d}$ before the recording session; animals monocularly deprived for shorter periods show the same effect (Crair et al., 1997). The effect on orientation selectivity of the second period of deprivation was quite different from that after the initial deprivation, in which the strong responses through the deprived eye were largely not orientation selective (Fig. $1 B, a-d$ ). After the reverse suture, optical CBIs were 0.376, 0.387, and 0.369 for kittens $\mathrm{K} 2, \mathrm{~K} 12$, and $\mathrm{K} 28$, respectively, indicating in all cases a predominant response to the eye ipsilateral to the cortex under study; that is, the OD (right) eye prevailed over the OND eye in its ability to elicit visual responses. The optical CBI in normal animals between P22 and P40 is 0.55 , and in animals deprived for 7 d starting around P28 it is 0.74 (Crair et al., 1997).

After completion of optical imaging, the activity of single units was recorded extracellularly in one or two vertical penetrations originating within the area that was mapped optically and directed down the medial bank of the lateral gyrus. Receptive fields of the neurons encountered were well within the binocular visual field. In Figure $2 A$, a sketch of electrode tracks along with the ocular dominance of the single units encountered is shown for each animal. Most of the neurons were dominated by the OD eye; only a few groups of neurons that were driven well or dominated by the OND eye were encountered along the electrode penetration.

Figure $2 B$ illustrates the ocular dominance distribution of the recorded neurons for each animal. In all cases after reverse suture, the OD eye has came to dominate the cortical response. The greatest and the smallest effects occurred in $\mathrm{K} 2$ and $\mathrm{K} 12$, respectively $(\mathrm{K} 2$ : $\mathrm{CBI}=0.16, \mathrm{RI}=0.84 ; \mathrm{K} 12$ : $\mathrm{CBI}=0.32, \mathrm{RI}=$ $0.75)$. However, in every case the OND eye still drove and dominated a small contingent of cortical neurons, in contrast with the almost complete absence of strong selective responses through the deprived eye in animals submitted to a single monocular deprivation (Fig. 2C) (Wiesel and Hubel, 1963; Movshon, 1976; Crair et al., 1997).

In summary, both optical and single-unit measures of cortical activity reveal a very substantial recovery of strong and selective responses to the OD eye and an accompanying reduction in response to the OND eye that is less severe and preserves greater selectivity than the deficit after the original deprivation.

\section{Organization of geniculocortical afferents}

\section{PHA-L injections into the LGN and cortical labeling}

In reverse-sutured animals, PHA-L injection sites were intended to be restricted to lamina A of both LGNs, to label selectively geniculocortical arbors in each hemisphere serving either the OD or the OND eye. Moreover, the injection sites had to be located in the binocular portion of the LGNs to ensure that the labeled afferents had been influenced by the binocular competitive interactions that are thought to underlie plastic remodeling (Hubel et al., 1977; LeVay et al., 1980). We obtained successful bilateral labeling of the geniculocortical connections in both hemispheres in only one animal (MUC 927, Table 2). In the other cases, only one hemisphere could be used, either because the quality of the label was not adequate to allow single axon reconstruction or because the injection site also involved lamina A1. In the normal P49 animal, PHA-L injection sites were also restricted to lamina A of the LGN. Therefore, all of the reconstructed arbors were in the pathway serving the contralateral eye. In kitten K28, one hemisphere was used for single arbor reconstructions, and the other was used for optical imaging and single-unit recordings.

The quality of labeling between the arbors reconstructed in reverse-sutured animals was similar to the normal animal, indicating that deprivation per se does not interfere with the transport of the lectin (Antonini and Stryker, 1996).

We refer to our reconstructions as arbors in layer IV and not as complete geniculocortical axons, because, as reported and discussed previously (Antonini and Stryker, 1996), PHA-L staining is very consistent in layer IV but becomes faint in the deeper portion of layer VI and the white matter, thus raising the possibility that collateralization of the main axonal trunk, had it occurred at this level, would have been missed. For these reasons, we have also disregarded the ramifications in layer VI.

Although labeled axons were found in both areas 17 and 18, we have exclusively analyzed geniculocortical afferents to area 17 . All of the reconstructed arbors were located in the top third of the medial bank of the lateral gyrus, in an area corresponding to the binocular zone of the visual cortex. This zone corresponds to that mapped by the electrode penetrations during single-unit recordings. Table 2 gives the experimental protocol and the list of arbors reconstructed in each animal.

\section{Geniculocortical arbors in a normal P49 kitten}

Five arbors were reconstructed from the medial bank of the lateral gyrus (area 17) in one P49 animal (Table 2). In Figure 3, three arbors $(N 1, N 3, N 6)$ are shown in coronal view and from the pial surface after a $90^{\circ}$ rotation along the ventrodorsal axis. The terminal arborizations of these arbors were characterized by a considerable extension along the ventrodorsal axis (reaching more than $2 \mathrm{~mm}$ in $\mathrm{N} 3$ ) and by the presence of zones of high concentration of collaterals. Overall, the P49 arbors were longer and more complex than those studied previously at P40, indicating that growth continues during this period. All arbors except N6 ramified mainly in the top portion of layer IV, with a few branches straddling the layer III/IV border. The terminal field of arbor N6 also covered deeper regions of layer IV.

\section{Geniculocortical arbors serving the $O D$ eye}

Thirteen geniculocortical arbors serving the OD eye were reconstructed from area 17 in kittens MUC 927 and K28 perfused at P48 and P49, respectively. These animals began $7 \mathrm{~d}$ of MD at age $\mathrm{P} 31 / 32$ and were reverse-sutured at P38/39 for $10 \mathrm{~d}$ (Table 2). This group of arbors varied greatly with respect to the complexity of their terminal arborization, as exemplified in Figure 4. The majority of arbors (arbors RS3, RS5, RS6, RS8, RS10, RS12, RS28, and RS31) appeared scantily ramified and generally occupied a restricted portion of layer IV, as is typical of arbors serving a deprived pathway (Antonini and Stryker, 1996). In contrast, five remaining arbors were large, like those in normal animals. Their 


\section{Reverse Suture}

\section{A Originally Deprived Eye}

a

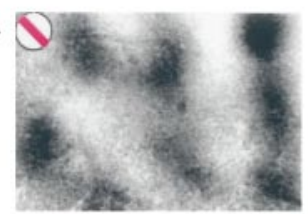

C

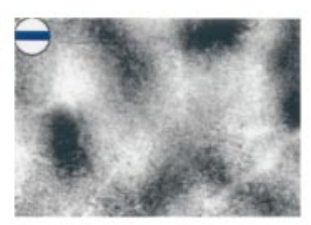

b

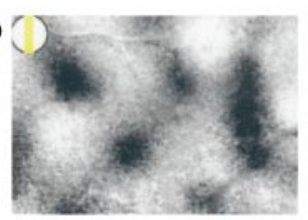

d

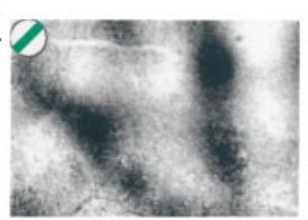

\section{Originally Non-deprived Eye}

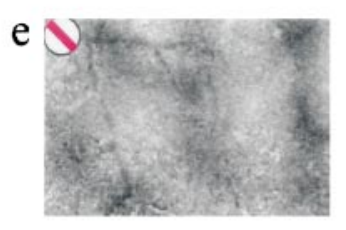

$\mathrm{g}$

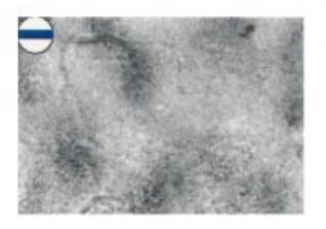

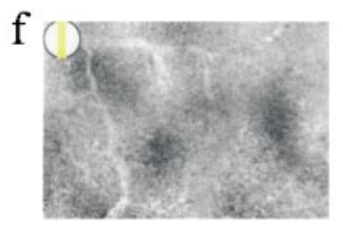

$\mathrm{h}$

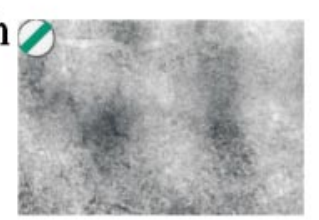

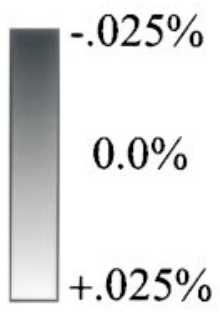
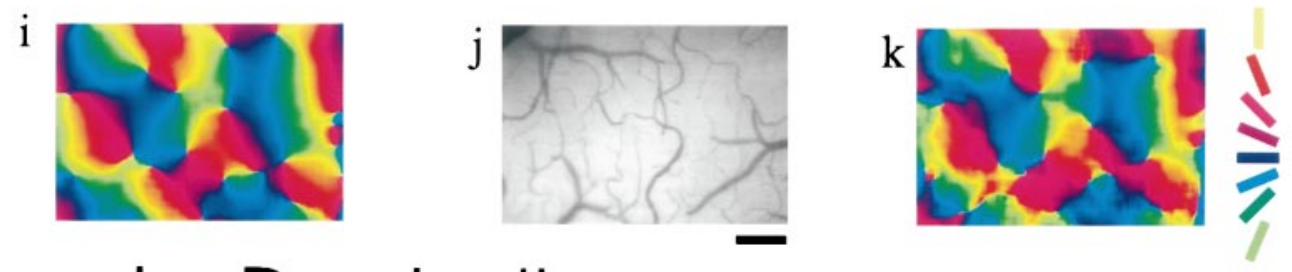

\section{Monocular Deprivation}

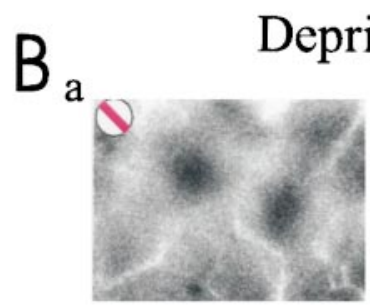

$\mathrm{b}$
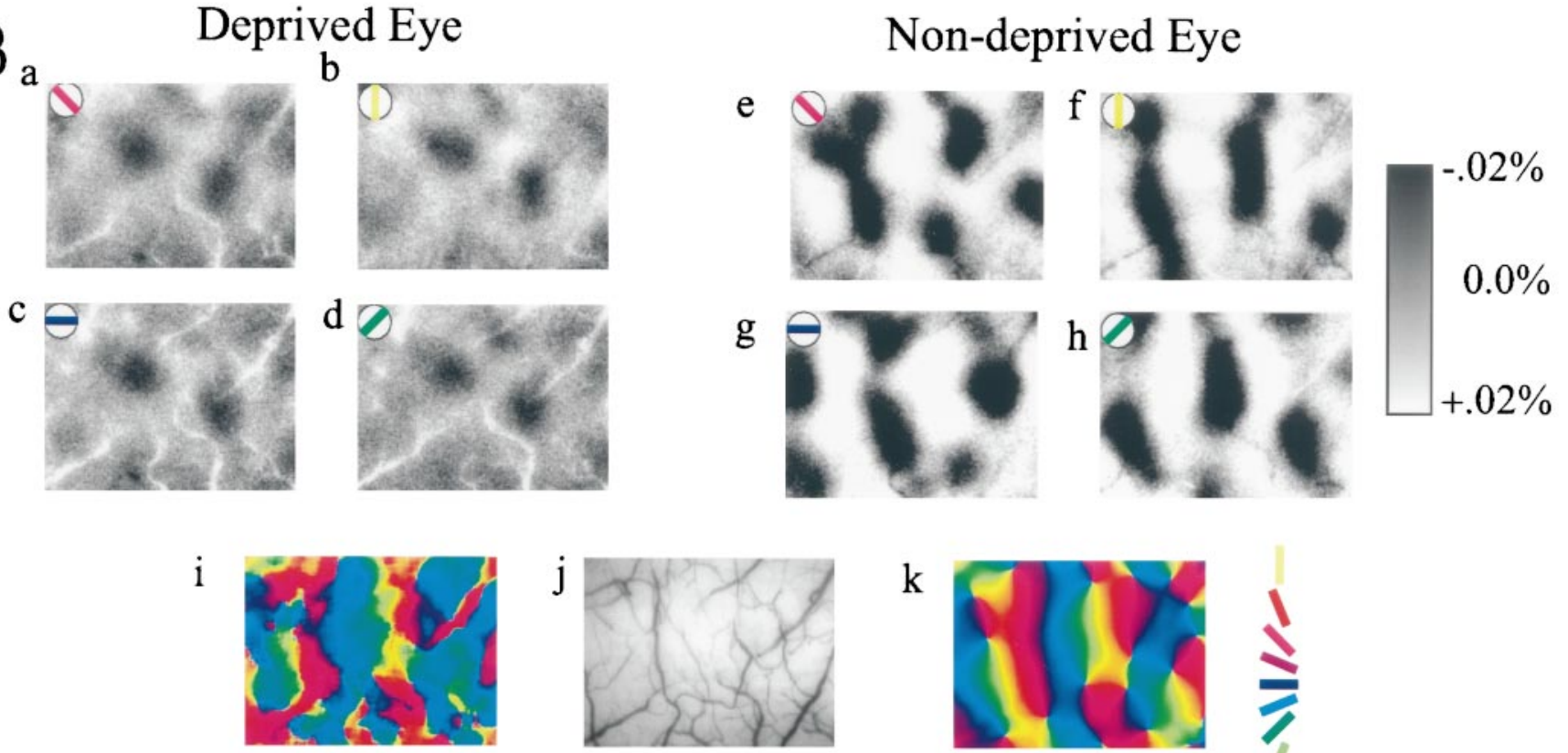

$\mathrm{k}$
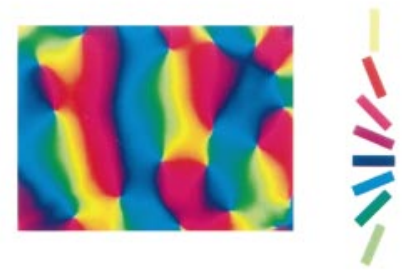

Figure 1. Optical imaging of intrinsic signals obtained from the lateral gyrus in a reverse-suture kitten $(A)$ and in a P38 kitten monocularly deprived for $7 \mathrm{~d}(B)$. In both $A$ and $B, a-h$ are cortical activity maps in response to each of the four oriented stimuli indicated in the left corner of each image; $j$ is the image of the vascular pattern, and $i$ and $k$ are color-coded angle maps of each hemisphere, combining the responses obtained from all orientations (see Materials and Methods). $A$, Distribution of cortical activity presented to the OD $(a-d)$ and the OND eye $(e-h)$. Note that the activity maps from the OND eye are orientation selective. $i, k$, Angle maps obtained from OD and OND eye, respectively. $B$, Distribution of cortical activity in response to oriented stimuli presented to the deprived eye $(a-d)$ and the nondeprived eye $(e-h) . i, k$, Angle maps obtained from deprived and nondeprived eye, respectively. Note in both the activity and angle maps that the responses through the deprived eye are nonoriented and limited to small cortical regions. Scale bar (shown in $A j$ for $A a-k, B a-k$ ): $500 \mu \mathrm{m}$. 


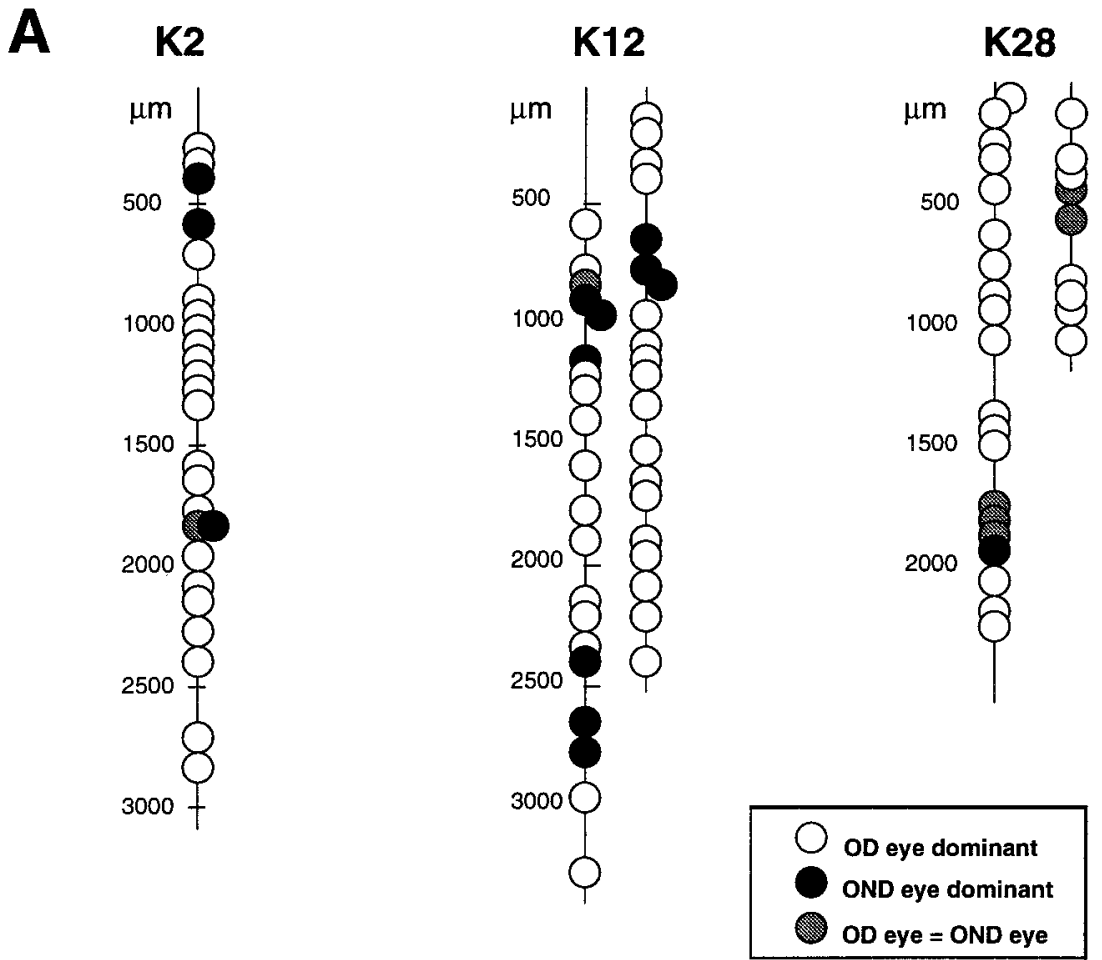

B
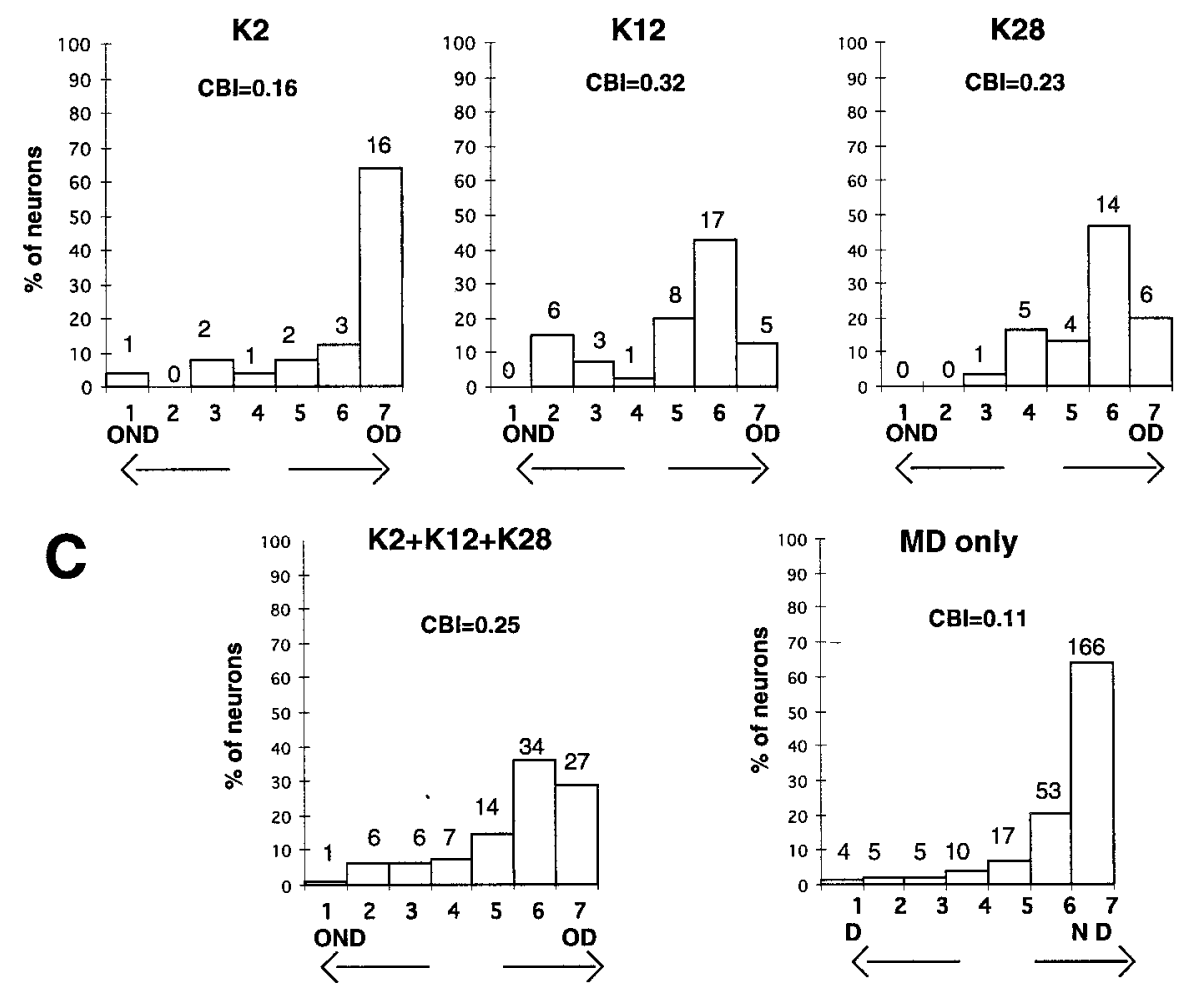

Figure 2. Single units recorded in vertical penetrations along the medial bank of the lateral gyrus in three reverse-sutured animals. $A$, Sketch of electrode tracks with the single units sequentially encountered is shown for each animal. The dominance of the OD or OND eye in activating each cell is indicated by different shading. Most neurons were dominated by the OD eye; units dominated by the OND eye, or equally driven by both eyes, were encountered in clusters. $B$, Ocular dominance distribution for neurons recorded in each animal. $C$, Ocular dominance distribution for the entire sample of cells recorded in the three reverse-sutured animals (left) and for 260 cells recorded in seven MD kittens [from Reiter and Stryker (1988)]. The contralateral bias index $(C B I)$ is indicated above each plot. terminal arborizations were extensively ramified and formed one or more clusters of densely packed collaterals, best appreciated in coronal view. Nine arbors ramified mainly in the top tier of layer IV, whereas the remaining four arbors spanned the entire width of layer IV. No relation was found between the complexity of the terminal arborization and its location within layer IV.
Geniculocortical arbors serving the OND eye

Fourteen arbors serving the OND eye were reconstructed from the medial bank of the lateral gyrus in three kittens (Table 2). Figure 5 illustrates four examples from this group of arbors. All but one of the arbors displayed the poorly ramified terminal arborization characteristic of deprived arbors. The exception was 


\begin{tabular}{|c|c|c|c|c|c|c|c|c|}
\hline \multirow[b]{2}{*}{ Animals } & \multicolumn{2}{|c|}{ First deprivation } & \multicolumn{2}{|c|}{ Second deprivation } & \multirow[b]{2}{*}{ Age at death } & \multirow{2}{*}{$\begin{array}{l}\text { Arbors serving } \\
\text { the OD eye }\end{array}$} & \multirow{2}{*}{$\begin{array}{l}\text { Arbors serving } \\
\text { the OND eye }\end{array}$} & \multirow{2}{*}{$\begin{array}{l}\text { Normal } \\
\text { arbors }\end{array}$} \\
\hline & Eye & Age & Eye & Age & & & & \\
\hline \multirow[t]{10}{*}{ MUC 927} & Left & P31 & Right & P38 & P48 & RS1 & RS13 & \\
\hline & & & & & & RS3 & RS14 & \\
\hline & & & & & & RS4 & RS15 & \\
\hline & & & & & & RS5 & RS16 & \\
\hline & & & & & & RS6 & RS17 & \\
\hline & & & & & & RS7 & RS18 & \\
\hline & & & & & & RS8 & RS19 & \\
\hline & & & & & & RS10 & & \\
\hline & & & & & & RS11 & & \\
\hline & & & & & & RS12 & & \\
\hline \multirow[t]{3}{*}{ K28 } & Right & $\mathrm{P} 32$ & Left & P39 & P49 & - RS28 & & \\
\hline & & & & & & RS30 & & \\
\hline & & & & & & RS31 & & \\
\hline \multirow[t]{2}{*}{ MUC 965} & Right & P35 & Left & P41 & P52 & & -RS20 & \\
\hline & & & & & & & RS21 & \\
\hline \multirow[t]{5}{*}{ MUC 976} & Right & $\mathrm{P} 34$ & Left & P41 & P51 & 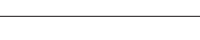 & -RS22 & \\
\hline & & & & & & & $\mathrm{RS} 23$ & \\
\hline & & & & & & & $\mathrm{RS} 24$ & \\
\hline & & & & & & & RS25 & \\
\hline & & & & & & & RS26 & \\
\hline \multirow[t]{5}{*}{ MUC 990} & \multirow{5}{*}{\multicolumn{3}{|c|}{ Nondeprived, control animal }} & & P49 & & & N1 \\
\hline & & & & & & & & $\mathrm{N} 2$ \\
\hline & & & & & & & & N3 \\
\hline & & & & & & & & N4 \\
\hline & & & & & & & & N6 \\
\hline
\end{tabular}

arbor RS14, which had a relatively elaborated terminal field that was also organized into two distinct clusters of collaterals separated by a collateral-free zone. All arbors except RS19 and RS31 ramified mainly in the top tier of layer IV.

\section{Quantitative aspects of geniculocortical arbors}

Previous experiments (Antonini and Stryker, 1993a, 1996) measured the remodeling of single geniculocortical arbors serving the deprived and nondeprived eye after 6-7 d of MD terminating at P40. The remodeling involved several aspects of the organization of the terminal arborization, including total length, complexity, maximal density, and characteristics of the areas of high density of collaterals. Reverse deprivation in the present experiment was planned to begin after $7 \mathrm{~d}$ of $\mathrm{MD}$ ending at P40, so that the data from previous experiments (Antonini and Stryker, 1996, 1998) would provide a baseline from which the further changes produced by reverse suture could be measured. In particular, for arbors serving the OD eye, the changes produced by reverse suture can be measured by comparison with the deprived arbors studied after 6-7 d MD (6/7d-D arbors). Similarly, the effects of reverse suture on OND arbors are evaluated by comparison with the nondeprived arbors after 6-7 d MD (6/7d-ND arbors). Finally, the deprived arbors are compared with those in normal animals.

The scattergrams in Figures 6 and 7 illustrate the values for the individual arbors in each experimental group. Table 3 lists for each parameter the median value for arbors in each experimental group, and Table 4 gives the significance of the statistical comparisons between groups (Mann-Whitney $U$ test).

\section{Total length and coverage area}

Measures of total length and coverage area reflect the size of geniculocortical arbors. The scattergrams in Figure $6 A, B$ show the distribution of these two parameters for arbors serving the OD and OND eye in reverse-sutured animals, for arbors serving the ND and D eye in animals monocularly deprived for 1 week $(6 / 7 d-N D$ and $6 / 7 d-D)$, and finally, for arbors reconstructed in normal animals at P30, P40, and P49.

Arbors serving the OD and the OND eye were significantly different from each other in size. For both parameters, the reverse opening of the eye caused a significant expansion of the arbors serving the OD eye compared with their state at the end of the first deprivation ( $p<0.01$; OD vs $6 / 7 \mathrm{~d}-\mathrm{D}$ ) (Table 4 ). The reverse MD caused an even greater reduction in the total length of arbors serving the OND eye as compared with their previous state after the initial week of monocular vision $(p<$ 0.001 ; OND vs $6 / 7 \mathrm{~d}-\mathrm{ND}$ ), although in this case the change in coverage area was not significant. The sizes of the deprived arbors at the end of the first and second period of deprivation were similar (not significant; OND vs 6/7d-D).

Comparisons with normal animals indicate that the reverse suture procedure interfered with normal development, rendering geniculocortical arbors smaller than arbors reconstructed in the age-matched control at P49. The opening of one eye during the second MD allowed OD arbors to only partially recover the total length, enough to make them similar to normal arbors at a younger age, P40 (OD vs Normal P40). 


\section{Normal Geniculocortical Arbors}

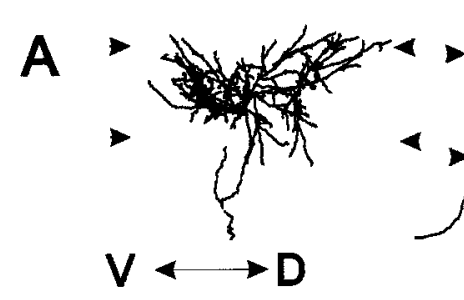

N1

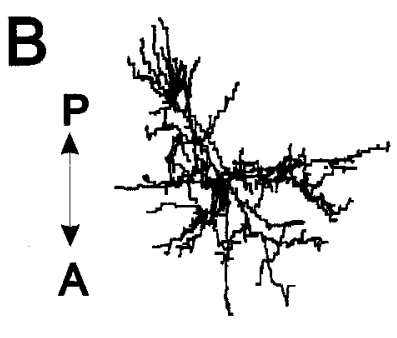

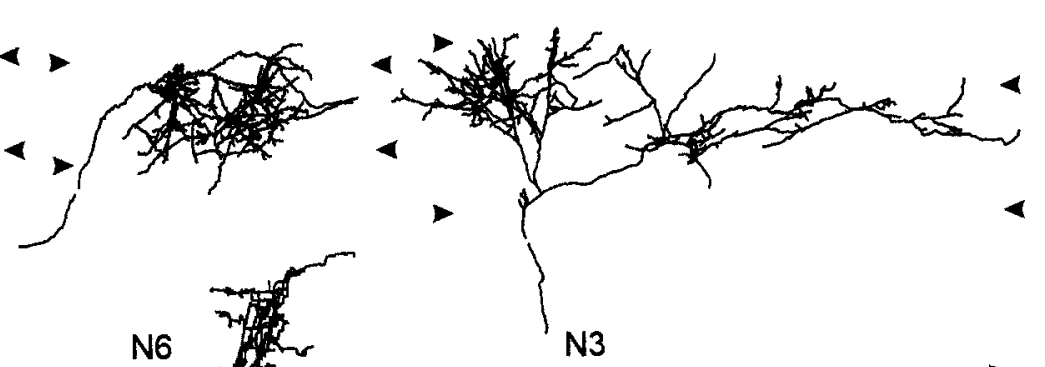

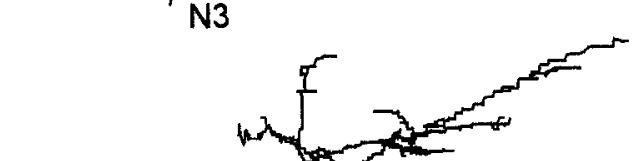

Figure 3. Computer reconstructions of PHA-L-immunostained axonal arbors in area 17 in a normal P49 animal. All geniculocortical arbors in normal, OND (Fig. 4), and OD (Fig. 5) animals were obtained from the dorsal-most portion of the medial bank of the lateral gyrus. $A$ shows the arbors as originally reconstructed in the coronal plane, and $B$ shows the arbors as seen from the pial surface, after a $90^{\circ}$ rotation along the dorsoventral axis of the lateral gyrus. The arrowheads indicate the boundaries of layer IV. $V \leftrightarrow D=$ ventrodorsal axis indicated in $A ; A \leftrightarrow P=$ anteroposterior axis indicated in $B$. Inset, Drawing of coronal section showing arbor N3 and rectangle in the medial bank of the lateral gyrus containing all arbors reconstructed. Inset: $D$, Dorsal direction; $V$, ventral direction.

\section{Originally Deprived Geniculocortical Arbors}

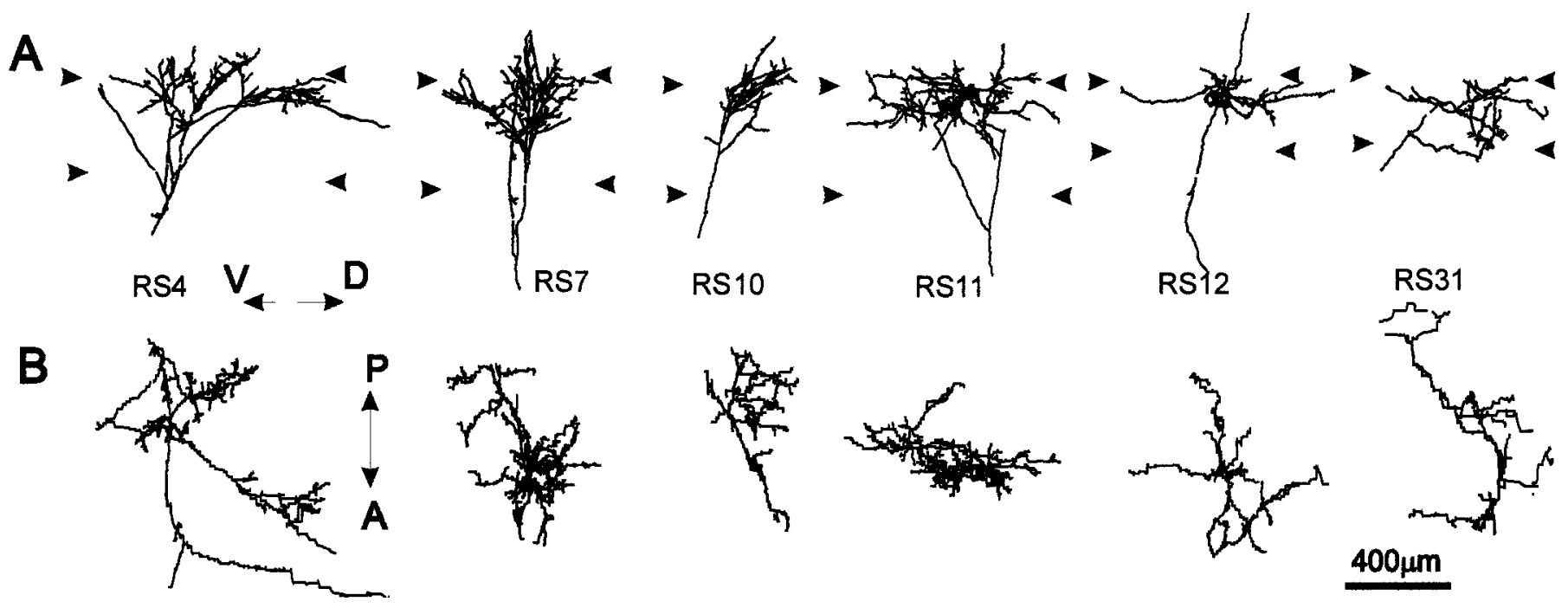

Figure 4. Computer reconstructions of PHA-L-immunostained geniculocortical arbors reconstructed in animals monocularly deprived for 1 week and reverse-sutured for $10 \mathrm{~d}$. These arbors serve the OD eye. Arbors are shown in coronal view $(A)$ and in surface view $(B)$. Abbreviations and symbols are the same as in Figure 3.

\section{Number of branch points and density measurements}

These parameters are an index of the complexity of the terminal arborization. The number of branch points and the maximal density of individual arbors in the different experimental groups are shown in Figure $7 A, B$; statistical comparisons are indicated in Table 4. After the reverse suture, OD arbors showed a significant increase in both measures relative to their state at the end of the first deprivation (OD vs 6/7d-D). Concurrently, after the second 


\section{Originally Non-Deprived Geniculocortical Arbors}
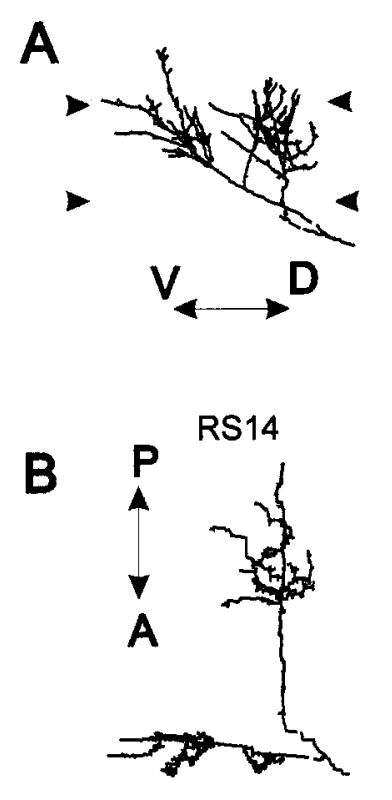

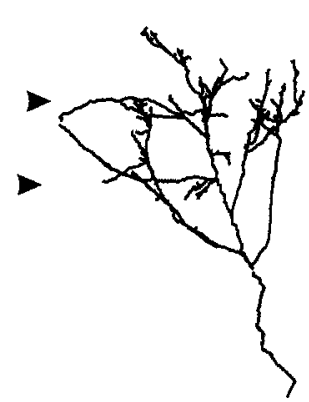

RS16

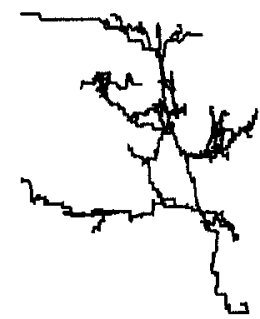

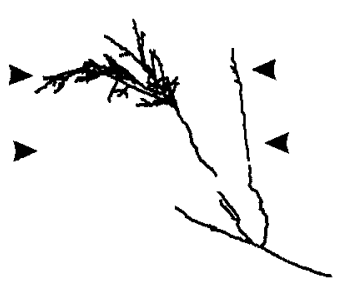

RS17

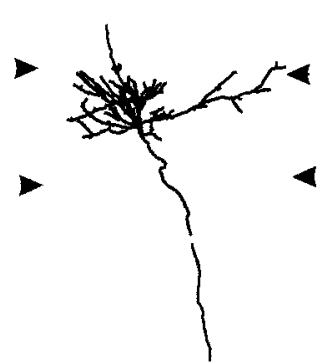

RS22

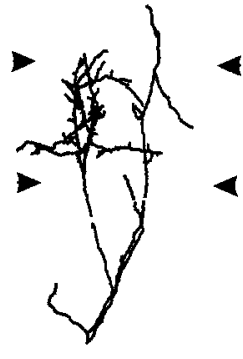

RS23

Figure 5. Computer reconstructions of PHA-L-immunostained geniculocortical arbors serving the OND eye reconstructed in reverse-sutured animals. Arbors are shown in coronal view $(A)$ and in surface view $(B)$. Abbreviations and symbols are the same as in Figure 3.

deprivation, OND arbors suffered a great reduction in the number of branch points $(p<0.001)$ and, to a lesser extent, in the maximal density $(p<0.04)$ compared with after the first deprivation (OND vs $6 / 7 \mathrm{~d}-\mathrm{ND})$.

At the end of the reverse suture, arbors serving both the OD and OND eye had many fewer branch points than arbors in the P49 age-matched control (OD vs P49, OND vs P49), although their maximal density was not different. Note, however, that the maximal density values of the population of normal arbors are very widespread.

Another approach for characterizing the internal structure of geniculocortical arbors is the assessment of regions of dense clustering of collateral branches within the terminal arborization. As discussed in Materials and Methods, the standard threshold density value was used to evaluate the zones of high-density areas in arbors of all groups of animals.

The scattergram of Figure $7 C$ indicates the total area of highdensity clusters for each arbor. Arbors reconstructed in normal animals at P40 and P49 show a great variability in the extent of the total area of high density. This measure is meaningful where dramatic changes occur but is not sensitive for detecting subtle modifications in the structure of the terminal arborization.

The terminal arborization of arbors serving the OND eye suffered a significant reduction in the total area of high density compared with their previous state at the end of the first deprivation (OND vs 6/7d-ND). Concomitantly, but to a lesser extent, the high-density areas in arbors serving the OD eye expanded after $10 \mathrm{~d}$ of monocular vision (OD vs 6/7d-D).

Note that the first deprivation was much more deleterious than the second in that many $6 / 7 \mathrm{~d}-\mathrm{D}$ arbors did not reach the defined threshold density of $38 \mu \mathrm{m} / 1000 \mu \mathrm{m}^{2}$ and thus did not form high-density areas (OND vs 6/7d-D). In contrast, reverse monoc- ular vision generally appeared to be sufficient to cause a reexpansion of the patches to the size they supposedly were $10 \mathrm{~d}$ before (OD vs 6/7d-ND). However, for a few arbors serving the OD eye, the area of the patches remained relatively small.

Comparison of effects of the first versus the reverse deprivation

With the aim of comparing the efficacy of the first MD versus the reverse MD in inducing plastic changes in geniculocortical arbors, we have measured the changes in each of the arbor parameters between the beginning and the end of each period of deprivation. Thus, for the first MD, we have measured the proportional changes of the arbors' parameters after 6-7 d of deprivation relative to values obtained in normal animals at an age corresponding to the beginning of the deprivation, i.e., between P30 and P40. Because the age at the beginning of the initial $7 \mathrm{~d}$ of deprivation was P33, we have interpolated a P33 value of each parameter from the normal values measured at P30 and P40 $(2 \times$ value at $\mathrm{P} 30+$ value at $\mathrm{P} 40) / 3$. For the reverse $\mathrm{MD}$, we have measured the changes of OD and OND arbors relative to arbors reconstructed in animals after the first deprivation, i.e., relative to arbors reconstructed after the 6/7 d MD. These results are plotted in Figure 8. For clarity, the percentage decrements or increments relative to the initial condition have been plotted as negative and positive values, respectively.

For deprived arbors, the first MD appears to be more deleterious than the reverse $\mathrm{MD}$ in inducing a reduction in the number of maximal density and high-density areas (Fig. $8 A$ ). The proportional changes in total length, branch points, and coverage area are similar in the two conditions.

In contrast, the open eye's arbors appear to benefit more from the reverse than from the first $\mathrm{MD}$, in that the increments of all 

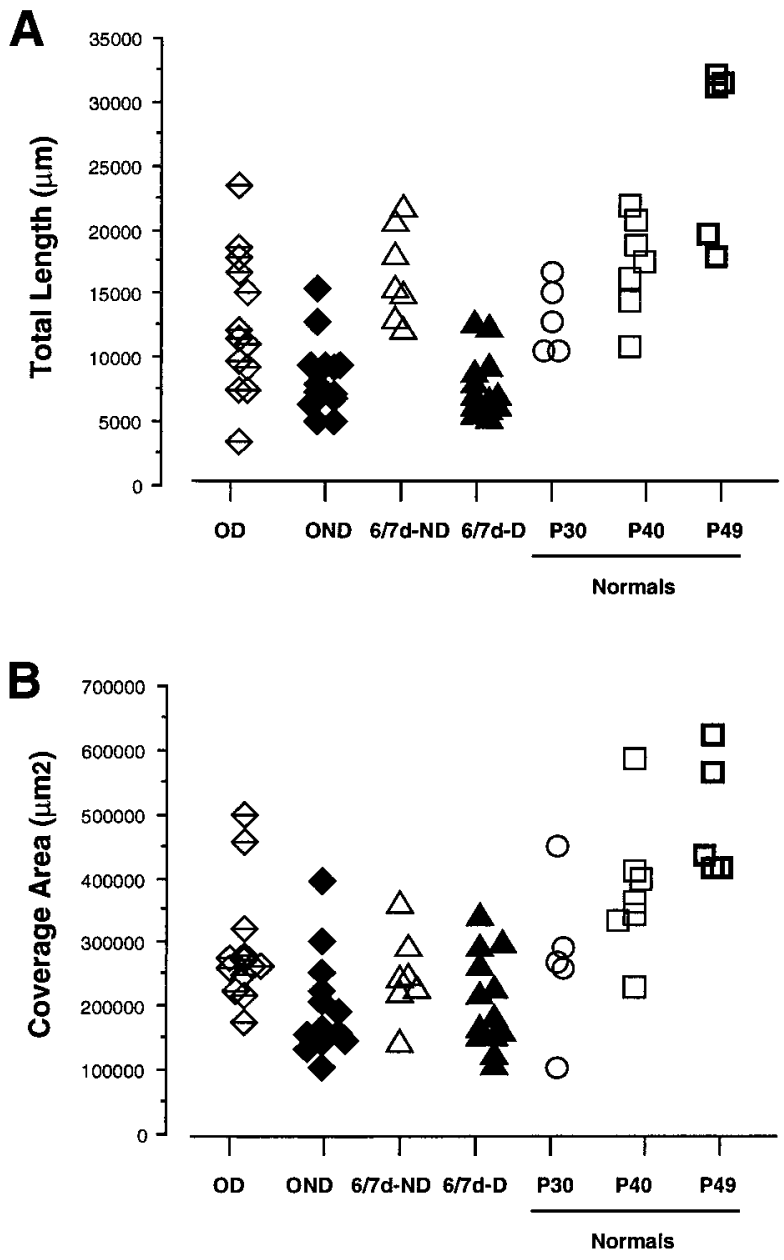

Figure 6. Scattergram of the total length $(A)$ and coverage area $(B)$ of the terminal arborization in layer IV (see Materials and Methods) for arbors reconstructed in reverse-sutured animals (OD and OND arbors) and in the P49 normal control. For comparison, data from arbors serving the deprived and nondeprived eye in animals monocularly deprived for 6-7 d (6/7d-D and 6/7d-ND arbors) and in normal animals at P30 and P40 are also plotted.

parameters relative to the initial condition are higher after the reverse MD than after the first MD.

\section{DISCUSSION}

The aim of this work was to analyze the extent to which the physiological recovery observed in the visual cortex after reverse $\mathrm{MD}$ is matched by changes in the anatomical characteristics of geniculocortical arbors serving the two eyes. This study was motivated by previous work on the effects of brief periods of MD on geniculocortical afferents (Antonini and Stryker, 1996). In that study we demonstrated that a few days of MD are sufficient to cause striking morphological changes in the geniculocortical connections serving the deprived eye, whereas arbors serving the nondeprived eye are little affected (Antonini and Stryker, 1996). These earlier findings suggested two conclusions. First, removal of axonal branches occurs faster than addition of new collaterals, at least during the first week of deprivation. Second, if the open eye's rapid ascent to dominance of cortical responses is predominantly the expression of geniculocortical innervation, then such dominance must be attributed to the loss of connections serving
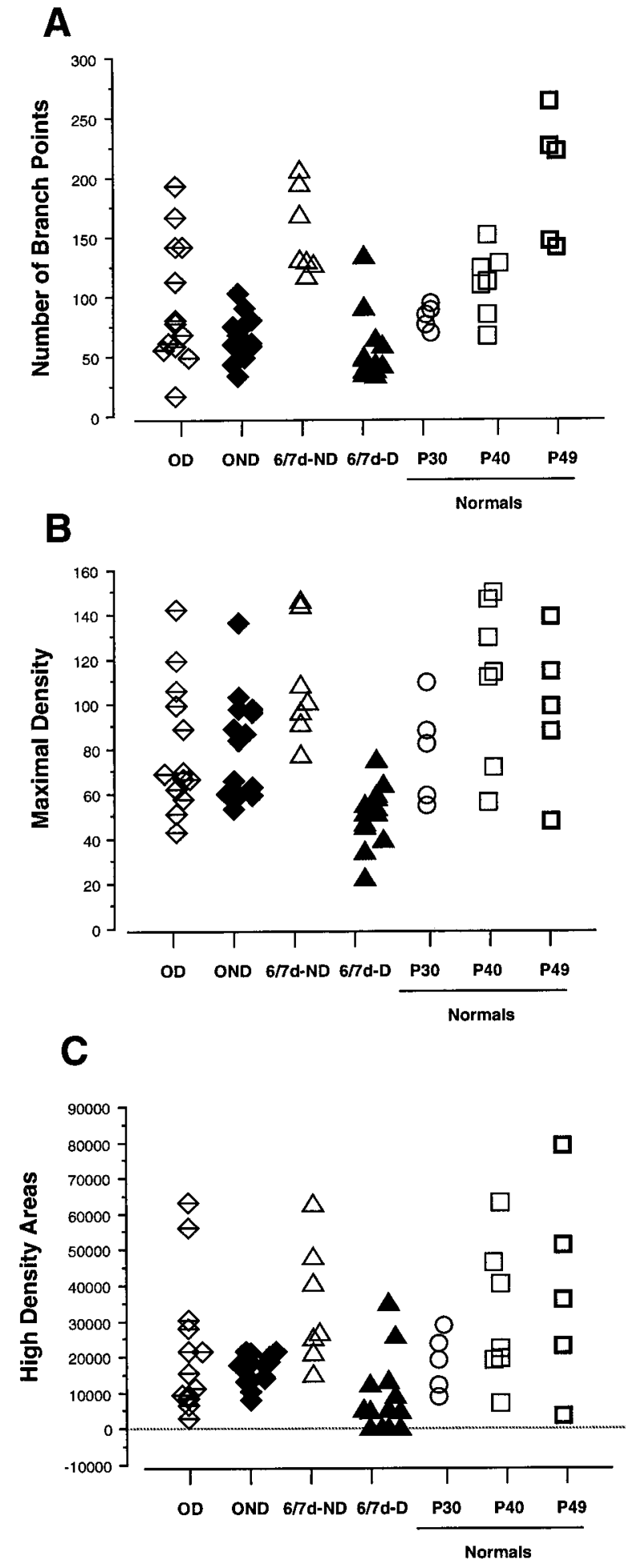

Figure 7. Scattergrams of number of branch points $(A)$, maximaldensity $(B)$, and $(C)$ high-density areas (see Materials and Methods) of the terminal arborization in layer IV in arbors reconstructed in reverse-sutured animals (OD and OND arbors), in animals monocularly deprived for $6-7 \mathrm{~d}(6 / 7 d-D$ and $6 / 7 d-N D$ arbors $)$, and in normal animals at P30, P40, and P49. The density is expressed in $\mu \mathrm{m} / 1000$ $\mu \mathrm{m}^{2}$. Note that the high-density areas in four $6 / 7 \mathrm{~d}-\mathrm{D}$ arbors are equal to 0 , because these arbors did not reach the threshold density of 38 $\mu \mathrm{m} / 1000 \mu \mathrm{m}^{2}$. 


\begin{tabular}{|c|c|c|c|c|c|c|c|}
\hline & & $\begin{array}{l}\text { Arbors } \\
(n)\end{array}$ & $\begin{array}{l}\text { Total length } \\
(\mu \mathrm{m})\end{array}$ & $\begin{array}{l}\text { Coverage area } \\
\left(\mu \mathrm{m}^{2}\right)\end{array}$ & $\begin{array}{l}\text { Branch points } \\
(n)\end{array}$ & $\begin{array}{l}\text { Maximal density } \\
\left(\mu \mathrm{m} / 1000 \mu \mathrm{m}^{2}\right)\end{array}$ & $\begin{array}{l}\text { High-density areas } \\
\left(\mu \mathrm{m}^{2}\right)\end{array}$ \\
\hline OD & P48-49 & 13 & 11582 & 264060 & 78 & 69 & 15336 \\
\hline OND & P48-52 & 14 & 7600 & 161406 & 62 & 85 & 17784 \\
\hline 6/7d-D & P40 & 13 & 6923 & 182016 & 43 & 51 & $8928^{a}$ \\
\hline 6/7d-ND & P40 & 7 & 15389 & 242064 & 131 & 101 & 26496 \\
\hline Normal & P30 & 5 & 12833 & 267696 & 85 & 82 & 19080 \\
\hline Normals & $\mathrm{P} 40$ & 7 & 17210 & 364068 & 113 & 114 & 22608 \\
\hline Normal & P49 & 5 & 31273 & 437400 & 222 & 99 & 36288 \\
\hline
\end{tabular}

${ }^{a}$ Total area of high density excluding the four arbors with no patches.

\begin{tabular}{|c|c|c|c|c|c|}
\hline & $\begin{array}{l}\text { Total length } \\
(\mu \mathrm{m})\end{array}$ & $\begin{array}{l}\text { Coverage area } \\
\left(\mu \mathrm{m}^{2}\right)\end{array}$ & $\begin{array}{l}\text { Branch points } \\
(n)\end{array}$ & $\begin{array}{l}\text { Maximal density } \\
\left(\mu \mathrm{m} / 1000 \mu \mathrm{m}^{2}\right)\end{array}$ & $\begin{array}{l}\text { High-density areas } \\
\left(\mu \mathrm{m}^{2}\right)\end{array}$ \\
\hline OD vs OND & $\uparrow 0.02$ & $\uparrow 0.003$ & ns & ns & ns \\
\hline OD vs $6 / 7 d-D$ & $\uparrow 0.01$ & $\uparrow 0.03$ & $\uparrow 0.02$ & $\uparrow 0.003$ & $\uparrow 0.02$ \\
\hline OND vs $6 / 7 d-N D$ & $\downarrow 0.001$ & ns & $\downarrow 0.001$ & $\downarrow 0.04$ & $\downarrow 0.004$ \\
\hline OD vs $6 / 7 d-N D$ & ns & ns & $\downarrow 0.03$ & $\downarrow 0.03$ & ns \\
\hline OND vs 6/7d-D & ns & ns & $\uparrow 0.05$ & $\uparrow 0.0002$ & $\uparrow 0.006$ \\
\hline OND vs normals P30 & $\downarrow 0.01$ & ns & $\downarrow 0.05$ & ns & ns \\
\hline OD vs normals P30 & ns & ns & ns & ns & ns \\
\hline OND vs normals P40 & $\downarrow 0.001$ & $\downarrow 0.002$ & $\downarrow 0.002$ & ns & $\downarrow 0.05$ \\
\hline OD vs normals $\mathrm{P} 40$ & ns & $\downarrow 0.05$ & ns & ns & ns \\
\hline OND vs normal P49 & $\downarrow 0.002$ & $\downarrow 0.002$ & $\downarrow 0.002$ & ns & ns \\
\hline OD vs normal P49 & $\downarrow 0.004$ & $\downarrow 0.01$ & $\downarrow 0.01$ & ns & $\mathrm{ns}$ \\
\hline
\end{tabular}

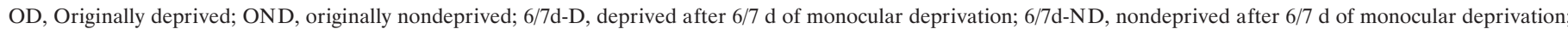

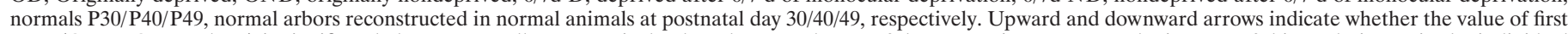

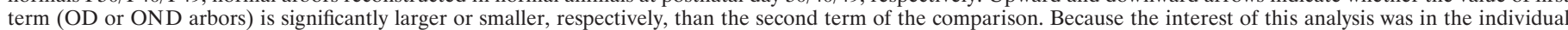

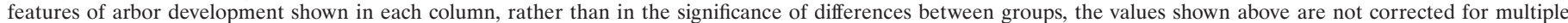

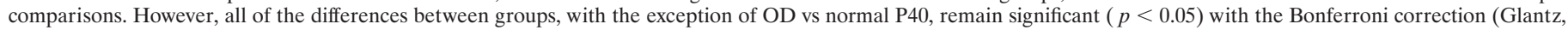
1992). ns, Not significant.

the deprived eye rather than to the expansion of connections serving the nondeprived eye.

To allow comparisons between data from the present reversesuture study and those from previous anatomical studies of the effects of brief MD, the reverse-suture protocol was designed to incorporate an initial period of deprivation identical to that studied previously (Antonini and Stryker, 1993a, 1996). Therefore, we were able to take the previous data as the baseline for changes produced by the reverse deprivation. The $10 \mathrm{~d}$ duration of the reverse MD was chosen to be consistent with protocols in the literature (Blakemore and van Sluyters, 1974; Movshon and Blakemore, 1974). According to these studies, $10 \mathrm{~d}$ of reverse suture in 6 week old kittens previously deprived for $10 \mathrm{~d}$ (van Sluyters, 1978) or since eye opening (Movshon, 1976) was sufficient to induce a reversal of the ocular dominance shift.

Because the shrinkage of deprived afferents is more rapid and more extensive than the expansion of those serving the open eye, we predicted that reverse suture from P40 to P50 near the end of the critical period might yield a cortex in which the pathways of both eyes were impaired. This prediction was confirmed. Indeed, we found that the OND arbors, whose baseline size at the end of the initial MD was similar to that of afferents in normal animals, did shrink rapidly and dramatically after the reverse suture, and the majority of the OD arbors did not greatly expand. Nonetheless, physiological responses to the OD eye recovered strength and selectivity, and responses to the OND eye, although diminished in strength, retained selectivity.

\section{Physiological and anatomical effects of reverse suture Cortical activity after reverse suture}

Binocular interactions in area 17 were studied by both optical imaging of intrinsic signals and single-unit recordings. Although the portions of the visual cortex examined by these two different techniques were different, one confined to the crown of the lateral gyrus and the other including its medial bank, both physiological measures were made within the central $10^{\circ}$ of the visual field, well within the most binocular zone of area 17. Ocular dominance is not reported to differ over this range of eccentricities. In agreement with previous studies (Movshon and Blakemore, 1974; Movshon, 1976), we have shown that after reverse suture, single-unit responses exhibit a shift in favor of the open, OD eye, implying that functionally the thalamocortical and/or intracortical circuits serving that eye have recovered substantially. In our experiments, the mean reversal index (see Materials and Methods) is 0.78 , very close to that found by Movshon (1976) after 9-12 d of reverse deprivation at the age of 5-6 weeks $(>0.70)$. Furthermore, the orientation specificity of the OD eye in the activity map of the cortex acquired during optical imaging is consistent with the recovery of function demonstrated by Kim and Bonhoeffer (1994), using the same experimental paradigm, and with the earlier single-unit findings of Van Sluyters (1978). The nearly complete functional recovery evident in neurophysiological experiments suggests that the reinnervation by the OD pathway ought to form in a manner mimicking the pattern of connectivity present before the first deprivation. 


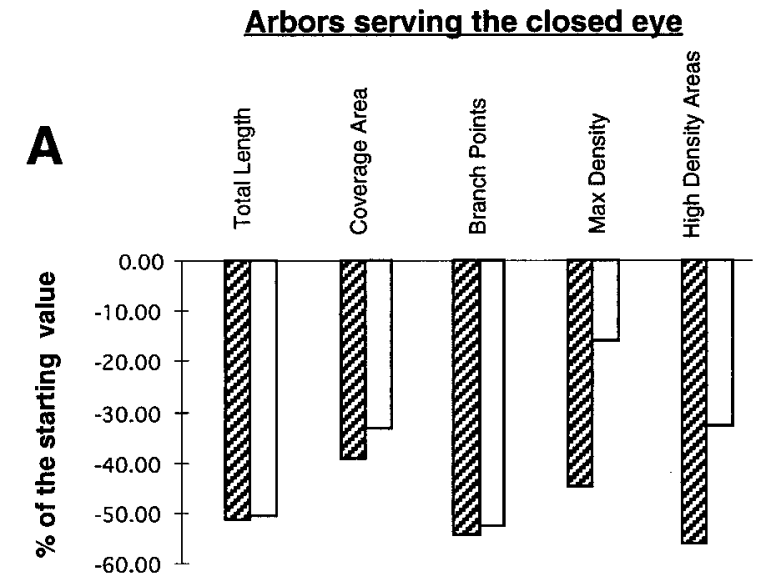

B

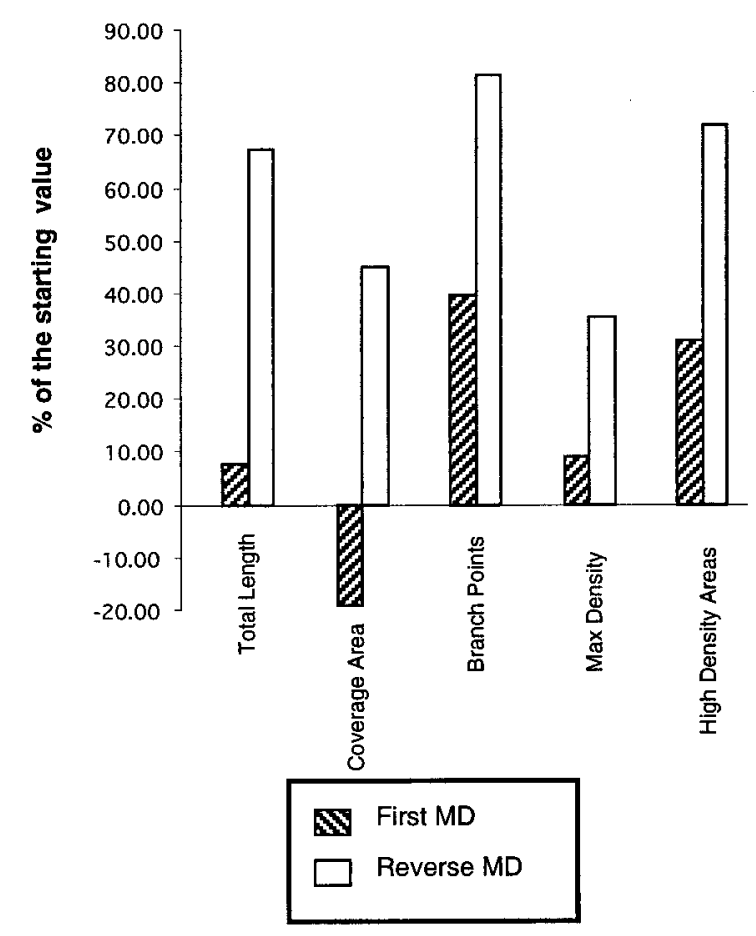

Figure 8. Percentage changes in total length, coverage area, number of branch points, maximal-density, and high-density areas after an initial period of 6-7 d of monocular deprivation (stipple bars) and after $10 \mathrm{~d}$ of reverse suture (white bars) in arbors serving the closed $(A)$ and the open $(B)$ eye. The percentage changes are referred to the state of the arbors before each deprivation. For the initial deprivation, comparisons were obtained relative to the values obtained in normal animals at P33 [(P33$6 / 7 d-D) \times 100 /$ P33 and $($ P33-6/7d- ND) $\times 100 /$ P33, for arbors serving the closed and open eye, respectively (see Results)]. For the reverse deprivation, we have measured the changes of OD and OND arbors relative to arbors reconstructed in animals deprived for 6-7 d [(6/7dNDOND $\times 100 / 6 / 7 d-N D$ and $(6 / 7 d-D-O D) \times 100 / 6 / 7 d-D$ for arbors serving the closed and open eye, respectively]. Note that reverse suture has a greater effect than the initial deprivation on arbors serving the open eye, whereas arbors serving the closed eye appear to be equally affected by both the initial and reverse deprivation.

The OND eye is typically considerably less potent than the newly opened eye in driving cortical neurons. However, this eye in reverse-sutured animals is still able to evoke strong cortical activity in clusters of neurons, in which it may even be dominant over the open eye. This observation is in contrast to that after the initial MD in which the majority of neurons become monocularly activated by the open eye. Single-unit recordings show that the reverse ocular dominance shift in our kittens is not as great as the shift at the end of the initial MD [contralateral bias index: 0.11 for the initial MD vs 0.25 for the reverse MD (Fig. 2C)]. The analysis of visual cortical activity by optical imaging also shows a substantial difference between the initial MD and reverse-sutured animals. In the initial MD, all of the residual strong activity elicited by the deprived eye is concentrated in cortical patches (centered on the pinwheel singularities of the orientation map) in which responses after MD are not orientation selective (Crair et al., 1997) (Fig. 1). These "deprived eye patches" of strong responses do not appear in the OND eye's map after reverse suture. Instead, the strongest cortical activity through the OND eye shows a clear selectivity for oriented stimuli.

Two factors may contribute to the stability of the orientation selectivity in the newly deprived pathway. First, the reverse deprivation occurs between P40 and P50, after the peak of the critical period, and therefore the visual cortical circuits serving the OND eye may inherently be less apt to collapse into patches of unselective responses. Second, the OND eye's pathway may not be pushed into such a collapse by competition from a strong pathway serving the other eye, because the other (OD) eye's pathway is itself largely collapsed at the outset of the reverse suture.

\section{Geniculocortical afferents after reverse suture}

Geniculocortical afferents were reconstructed from the medial bank of the lateral gyrus, in approximately the same portion of area 17 from which single-unit recordings were made. After reverse suture, we found a significant restructuring of single afferents of OD and OND arbors relative to their condition after the first MD. Specifically, the OND arbors suffer a reduction of almost all the parameters studied, with the result that they are actually smaller than normal arbors at P50, their age-matched controls. Thus, as with an initial MD, reverse deprivation is effective in inducing a loss of terminals in the deprived arbors. However, the morphological condition of arbors serving the OND eye after the reverse MD is not as deficient as after the first $\mathrm{MD}$; indeed, OND arbors appear on average larger and more complex than the 6/7d-D group. These findings are consistent with our observations from the physiological results discussed above. Again, this may be attributable to the slight advantage that the OND arbors acquired during the first deprivation (Antonini and Stryker, 1996), or it may be purely age-dependent, reflecting the decreased efficacy of plastic processes toward the end of the critical period.

The group of OD arbors shows an increase in all parameters studied. However, this group of arbors is quite heterogeneous. Ten days of monocular usage of the OD eye has clearly promoted regrowth of some arbors, whereas others still maintain characteristics of deprived arbors. Thus, regrowth of terminals can occur within a short period of reverse suture, but it is not a widespread phenomenon. We can only speculate which population of geniculocortical arbors is more predisposed to regrowth of terminals. For example, terminals located in the periphery of ocular dominance columns might be more susceptible to the influences of competitive interactions between the two eyes. After reverse suture, OD arbors are still smaller than normal age-matched controls. It is possible that a longer period of reverse suture would allow the OD arbors to recover fully. 
Earlier works have suggested anatomical reorganizations after reverse suture. For example, Dursteler et al. (1976) compared neuronal somata sizes in the OD and OND laminae of the lateral geniculate nucleus. They inferred a partial regrowth by cells in the OD lamina and a concomitant shrinkage of cells in the OND lamina after only a few days of reverse suture. In the monkey, transneuronal labeling after reverse suture during the critical period shows a recovery of the OD eye's lost territory in layer IV (LeVay et al., 1980; Swindale et al., 1981), also consistent with the present findings. These earlier findings are informative about the relative sizes and territories occupied by the two eyes' pathways, but they do not allow the absolute comparisons with geniculocortical arbors in age-matched normal control animals.

\section{Comparison of anatomy and physiology with behavior}

The present findings show only a partial correlation between physiological and morphological changes after reverse suture, with the morphology of both eyes' pathways more impaired than one would expect from the physiology. Strong physiological responses through the OD eye are not fully matched by the morphological characteristics of the OD afferents, because only a few appear to have regrown. The OND afferents are also less well developed morphologically than the physiological responses would suggest, because these afferents appear to be nearly as affected as deprived-eye afferents after the initial MD, but the cortical activity elicited by this eye is stronger and very much more selective than that after an initial MD.

These findings indicate that the substrate for the reacquisition of apparently normal responses through the OD eye is not, as one might have predicted, the reestablishment of the pattern of connectivity that was present before the onset of any deprivation (Kim and Bonhoeffer, 1994). Reconstituted responses that mimic the original ones can be generated by afferent input that is different in detail and considerably impoverished. This finding would suggest that intracortical circuitry may be more stable than the thalamocortical input and can organize and maintain strong and selective responses when this input is impoverished. Strong responses to weak afferent input may be generated by intracortical circuitry through several mechanisms. For example, an imbalance of reciprocal inhibitory influences among cortical neurons located in different ocular dominance columns (Ramoa et al., 1988; Mower and Christen, 1989; Sengpiel et al., 1994) might enhance the small advantage of the OD pathway and increase its cortical representation. In addition, feed-forward excitation or amplification within a cortical column could also enhance weak input signals (Douglas et al., 1995).

This view of stable intracortical connectivity appears to contradict the idea that the rapid plasticity of upper layer cortical responses after brief MD depends on rapid changes in corticocortical rather than thalamocortical circuitry (Crair et al., 1997). One may reconcile this apparent contradiction by assuming that it is the pattern of corticocortical connections that is responsible for the selective responses that we have measured and that this pattern is stable, but that the activation of these intracortical circuits depends on the relative strengths of inputs from the two eyes. Thus, when inputs from both eyes are reduced, the selective cortical circuits can respond to both.

Behaviorally, the recovery from the effects of deprivation after reverse occlusion is not stable (Murphy and Mitchell, 1986, 1987). Animals initially deprived from eye-opening to 4 or 5 weeks, followed by a period of reverse suture and then binocular vision, develop a severe bilateral amblyopia. In these animals, the acuity of the OD eye drops dramatically to very low levels, independent of the length of the reverse MD. Vision in the OND eye recovers somewhat but never reaches normal acuity (Mitchell, 1991). Such binocular amblyopia does not occur after binocular recovery from an initial MD. These findings suggest that the impaired morphology of both eyes' pathways after reverse suture may have behavioral consequences that could not be predicted from the present physiological changes.

\section{REFERENCES}

Antonini A, Stryker MP (1993a) Rapid remodeling of axonal arbors in the visual cortex. Science 260:1819-1821.

Antonini A, Stryker MP (1993b) Development of individual geniculocortical arbors in cat striate cortex and effects of binocular impulse blockade. J Neurosci 13:3549-3573.

Antonini A, Stryker MP (1996) Plasticity of geniculocortical afferents following brief or prolonged monocular occlusion in the cat. J Comp Neurol 369:64-82.

Antonini A, Stryker MP (1998) Effect of sensory disuse on geniculate afferents to cat visual cortex. Vis Neurosci 15:401-409.

Blakemore C, Hawken MJ (1982) Rapid restoration of functional input to the visual cortex of the cat after brief monocular deprivation. J Physiol (Lond) 327:463-487.

Blakemore C, van Sluyters RC (1974) Reversal of the physiological effects of monocular deprivation in kittens: further evidence for a sensitive period. J Physiol (Lond) 237:195-216.

Blakemore C, Garey LJ, Vital-Durand F (1978) The physiological effects of monocular deprivation and their reversal in the monkey's visual cortex. J Physiol (Lond) 283:223-262.

Blakemore C, Vital-Durand F, Garey LJ (1981) Recovery from monocular deprivation in the monkey. I. Reversal of physiological effects in the visual cortex. Proc R Soc Lond B Biol Sci 213:399-423.

Chow KL, Stewart DL (1972) Reversal of structural and functional effects of long-term visual deprivation in cats. Exp Neurol 34:409-433.

Crair MC, Ruthazer ES, Gillespie DC, Stryker MP (1997) Relationship between the ocular dominance and orientation maps in visual cortex of monocularly deprived cats. Neuron 9:307-318.

Crair MC, Gillespie DC, Stryker MP (1998) The role of visual experience in the development of columns in cat visual cortex. Science 279:566-570.

Douglas RJ, Koch C, Mahowald M, Martin KA, Suarez HH (1995) Recurrent excitation in neocortical circuits. Science 269:981-985.

Dursteler MR, Garey LJ, Movshon JA (1976) Reversal of the morphological effects of monocular deprivation in the kitten's lateral geniculate nucleus. J Physiol (Lond) 261:189-210.

Freeman RD, Mallach R, Hartley S (1981) Responsivity of normal kitten striate cortex deteriorates after brief binocular deprivation. J Neurophysiol 45:1074-1084.

Fregnac Y, Imbert M (1984) Development of neuronal selectivity in primary visual cortex of cat. Physiol Rev 64:325-434.

Gerfen CR, Sawchenko PE (1984) An anterograde neuroanatomical tracing method that shows the detailed morphology of neurons, their axons and terminals: immunohistochemical localization of an axonally transported plant lectin, Phaseolus vulgaris leucoagglutinin (PHA-L). Brain Res 290:219-238.

Giffin F, Mitchell DE (1978) The rate of recovery of vision after early monocular deprivation in kittens. J Physiol (Lond) 274:511-537.

Glantz SA (1992) Primer of biostatistics, Ed 3. New York: McGraw-Hill.

Hubel DH, Wiesel TN (1962) Receptive fields, binocular interactions, and functional architecture in the cat's visual cortex. J Physiol (Lond) 160:106-154.

Hubel DH, Wiesel TN (1970) The period of susceptibility to the physiological effects of unilateral eye closure in kittens. J Physiol (Lond) 206:419436.

Hubel DH, Wiesel TN, LeVay SM (1977) Plasticity of ocular dominance columns in monkey striate cortex. Philos Trans R Soc Lond B Biol Sci 278:377-409.

Kim DS, Bonhoeffer T (1994) Reverse occlusion leads to precise restoration of orientation preference maps in kitten visual cortex. Nature 370:370-372.

LeVay SM, Wiesel TN, Hubel DH (1980) The development of ocular dominance columns in normal and visually deprived monkeys. J Comp Neurol 191:1-51. 
Malach R, Ebert R, van Sluyters RC (1984) Recovery from effects of brief monocular deprivation in the kitten. J Neurophysiol 51:538-551.

Mitchell DE (1991) The long-term effectiveness of different regimens of occlusion on recovery from early monocular deprivation in kittens. Philos Trans R Soc Lond B Biol Sci 333:51-79.

Mitchell DE, Cynader M, Movshon JA (1977) Recovery from the effects of monocular deprivation in kittens. J Comp Neurol 176:53-63.

Movshon JA (1976) Reversal of the physiological effects of monocular deprivation in the kitten's visual cortex. J Physiol (Lond) 261:125-174.

Movshon JA, Blakemore C (1974) Functional reinnervation in kitten visual cortex. Nature 251:504-505.

Movshon JA, Dursteler MR (1977) Effects of brief periods of unilateral eye closure on the kitten's visual system. J Neurophysiol 40:1255-1265.

Mower GD, Christen WG (1989) Evidence for an enhanced role of GABA inhibition in visual cortical ocular dominance of cats reared with abnormal monocular experience. Dev Brain Res 45:211-218.

Murphy KM, Mitchell DE (1986) Bilateral amblyopia after a short period of reverse occlusion in kittens. Nature 323:536-538.

Murphy KM, Mitchell DE (1987) Reduced visual acuity in both eyes of monocularly deprived kittens following a short or long period of reverse occlusion. J Neurosci 7:1526-1536.

Olson CR, Freeman RD (1977) Monocular deprivation and recovery during the sensitive period in kittens. J Neurophysiol 41:65-74.

Passera A, Fulks S, Schneider GE, Ayres S, Jhaveri S, Erzurumlu RS (1988) The M.I.T. "Neurotrace" system for microcomputer-aided microscopy. Soc Neurosci Abstr 14:550.

Ramoa AS, Paradiso MA, Freeman RD (1988) Blockade of intracortical inhibition in kitten striate cortex: effects on receptive field properties and associated loss of ocular dominance plasticity. Exp Brain Res 73:285-296.

Reiter HO, Stryker MP (1988) Neural plasticity without postsynaptic action potentials: less-active inputs become dominant when kitten visual cortical cells are pharmacologically inhibited. Proc Natl Acad Sci USA 85:3623-3627.

Sengpiel F, Blakemore C, Kind PC, Harrad R (1994) Interocular suppression in the visual cortex of strabismic cats. J Neurosci 14:6855-6871.

Shatz CJ, Stryker MP (1978) Ocular dominance in layer IV of the cat's visual cortex and the effects of monocular deprivation. J Physiol (Lond) 281:267-283.

Sherman SM, Spear PD (1982) Organization of visual pathways in normal and visually deprived cats. Physiol Rev 62:738-855.

Swindale NV, Vital-Durand F, Blakemore C (1981) Recovery from monocular deprivation in the monkey. III. Reversal of anatomical effects in the visual cortex. Proc R Soc Lond B Biol Sci 213:435-450.

van Sluyters RC (1978) Reversal of the physiological effects of brief periods of monocular deprivation in the kitten. J Physiol (Lond) 284:1-17.

Wiesel TN, Hubel DH (1963) Single-cell responses in striate cortex of kittens deprived of vision in one eye. J Neurophysiol 26:1003-1017.

Wiesel TN, Hubel DH (1965) Comparison of the effects of unilateral and bilateral eye closure on cortical unit responses in kittens. J Neurophysiol 28:1029-1040. 